\title{
Detection of an inversion in the $T y-2$ region between $S$. lycopersicum and $S$. habrochaites by a combination of de novo genome assembly and BAC cloning
}

\author{
Anne-Marie A. Wolters ${ }^{1} \cdot$ Myluska Caro $^{1} \cdot$ Shufang Dong $^{2} \cdot$ Richard Finkers $^{1}$ • \\ Jianchang Gao ${ }^{2} \cdot$ Richard G. F. Visser $^{1} \cdot$ Xiaoxuan Wang ${ }^{2}$ Yongchen Du ${ }^{2}$. \\ Yuling Bai ${ }^{1}$
}

Received: 11 April 2015 / Accepted: 13 June 2015 / Published online: 8 July 2015

(C) The Author(s) 2015. This article is published with open access at Springerlink.com

\begin{abstract}
Key message A chromosomal inversion associated with the tomato $T y-2$ gene for TYLCV resistance is the cause of severe suppression of recombination in a tomato $T y-2$ introgression line.

Abstract Among tomato and its wild relatives inversions are often observed, which result in suppression of recombination. Such inversions hamper the transfer of important traits from a related species to the crop by introgression breeding. Suppression of recombination was reported for the TYLCV resistance gene, $T y-2$, which has been introgressed in cultivated tomato (Solanum lycopersicum) from the wild relative $S$. habrochaites accession B6013. Ty-2 was mapped to a $300-\mathrm{kb}$ region on the long arm of chromosome 11. The suppression of recombination in the Ty-2 region could be caused by chromosomal rearrangements in S. habrochaites compared with S. lycopersicum. With the aim of visualizing the genome structure of the
\end{abstract}

Communicated by H.-Q. Ling.

A.-M. A. Wolters, M. Caro and S. Dong equally contributed to this work.

Electronic supplementary material The online version of this article (doi:10.1007/s00122-015-2561-6) contains supplementary material, which is available to authorized users.

Yuling Bai

bai.yuling@wur.nl

1 Wageningen UR Plant Breeding, Wageningen University \& Research Centre, P.O. Box 386, 6700 AJ Wageningen, The Netherlands

2 The Institute of Vegetables and Flowers, Chinese Academy of Agricultural Sciences, Zhongguancunnandajie 12, Beijing 100081, People's Republic of China
Ty-2 region, we compared the draft de novo assembly of $S$. habrochaites accession LYC4 with the sequence of cultivated tomato ('Heinz'). Furthermore, using populations derived from intraspecific crosses of $S$. habrochaites accessions, the order of markers in the $T y-2$ region was studied. Results showed the presence of an inversion of approximately $200 \mathrm{~kb}$ in the $T y-2$ region when comparing S. lycopersicum and $S$. habrochaites. By sequencing a BAC clone from the $T y-2$ introgression line, one inversion breakpoint was identified. Finally, the obtained results are discussed with respect to introgression breeding and the importance of a priori de novo sequencing of the species involved.

\section{Introduction}

In genetics, introgression (also known as introgressive hybridization) is the transfer of a gene from one species into the gene pool of another species. Such a transfer starts with an interspecific hybridisation and is followed by backcrossings with one of the parental species. In breeding, introgression is an important strategy to broaden the genetic base of highly inbred crops such as tomato by transferring economically important traits from a related species to the crop. One of the major problems in introgression breeding is caused by chromosomal rearrangements, such as inversions and translocations, between the donor species and the crop (Szinay et al. 2010). Genetic maps created from different intraspecific or interspecific crosses using the same markers can indicate co-linearity or a change in order of markers in distinct chromosomal regions. In the Solanaceae family, genetic maps have been used to detect chromosomal rearrangements (e.g. Wu and Tanksley 2010; Doğanlar et al. 2014). Also, the study of pachytene synaptonemal complexes in interspecific $\mathrm{F}_{1}$ hybrids can indicate 
the presence of chromosomal rearrangements (Anderson et al. 2010). Meanwhile, cross-species BAC fluorescence in situ hybridisation (FISH) analysis has been shown to be a powerful instrument to identify chromosomal rearrangements in the Solanaceae family (van der Knaap et al. 2004; Iovene et al. 2008; Tang et al. 2008) and more specifically, among related species of Solanum (Lou et al. 2010; Verlaan et al. 2011; Peters et al. 2012; Szinay et al. 2012; Shearer et al. 2014). In introgression breeding this technology can be used as a diagnostic tool to monitor meiotic disturbances in the pairing of homoeologous chromosomes from crops and their related species. Nowadays, the released full genome sequences of closely related species have facilitated comparative genome analysis. Occurrences of both large-scale and small-scale rearrangements have been reported between tomato and potato genomes (The Potato Genome Sequencing Consortium 2011; The Tomato Genome Consortium 2012). For a co-linearity study of two genomes, a reference genome sequence should not only be available for the cultivated species, but also for the wild donor species. To this end, de novo assembly of genome sequences of three wild relatives of tomato has been undertaken recently (The 100 Tomato Genome Sequencing Consortium 2014).

Among tomato and its wild relatives, inversions are often observed (Szinay et al. 2012) which can cause meiotic pairing disturbances between homoeologues. Crossovers are unlikely to occur in the inverted region, which results in suppression of recombination (Szinay et al. 2010). Thus, the inverted region will be genetically inherited as one locus during the introgression and many unwanted sequences in the inverted region will be transferred together with the gene of interest from the wild donor to the crop species, a phenomenon known as linkage drag. A good example is the $T y-1$ gene which originated from $S$. chilense and confers resistance to Tomato yellow leaf curl virus (TYLCV) (Verlaan et al. 2013). Chromosomal rearrangements between $S$. chilense and the cultivated tomato were detected by BAC-FISH (Verlaan et al. 2011). These rearrangements caused severe suppression of recombination in the $T y-1$ region and thus hampered the $T y-1$ introgression (Verlaan et al. 2011). Suppression of recombination was reported for another TYLCV resistance gene, $T y-2$, which has been introgressed in cultivated tomato (Solanum lycopersicum) from the wild relative S. habrochaites accession B6013 (Kalloo and Banerjee 1990). The gene has been mapped on the long arm of chromosome 11 (Hanson et al. 2000; Ji et al. 2009), and fine mapped to a 300-kb region (Yang et al. 2014). Attempts to clone the gene have been hampered by the occurrence of severe suppression of recombination in a large part of this region. Such suppression of recombination could be caused by chromosomal rearrangements in S. habrochaites compared with $S$. lycopersicum, as shown previously for the $T y-1$ gene (Verlaan et al. 2011). However, for the $T y$ 2 region, FISH on pachytene chromosomes using three BACs spanning the introgression region and one BAC outside the region resulted in overlapping fluorescing signals (Yang et al. 2014). Because of this, the order of the BACs could not be determined, and therefore no conclusion could be drawn on the cause of the suppression of recombination.

In order to visualize the genome structure of the $T y-2$ region, in this study we combined de novo genome assembly and BAC cloning. First, the draft de novo assembly of $S$. habrochaites accession LYC4 was compared with the genome sequence of cultivated tomato ('Heinz') to determine whether a chromosomal rearrangement has occurred in the $T y-2$ region. Secondly, BAC cloning of the $T y-2$ introgression line was performed. Furthermore, recombinant screening of $F_{2}$ populations derived from intraspecific crosses of $S$. habrochaites accessions was carried out. Taken together, the results showed the presence of an inversion of approximately $200 \mathrm{~kb}$ in the $T y-2$ region when comparing S. lycopersicum and S. habrochaites.

\section{Materials and methods}

\section{Plant materials and DNA isolation}

Solanum lycopersicum 'Moneymaker' (MM), S. habrochaites accessions LYC4, G1.1560 (=CGN15790), G1.1257 (=CGN15370), G1.1606 (=CGN24036) and G1.1290 (=CGN15391) were obtained from the Centre for Genetic Resources (CGN), Wageningen, the Netherlands.

$\mathrm{F}_{2}$ family PV95279 was obtained from an interspecific cross between $S$. lycopersicum MM and S. habrochaites accession G1.1257. $F_{2}$ families of intraspecific crosses between S. habrochaites accessions were PV960350 (G1.1257 × G1.1560), PV960357 (G1.1560 x G1.1606) and PV970303 (G1.1290 × G1.1560). Seeds from the $F_{2}$ populations and parental accessions were sown in plastic cell trays and kept in a germination chamber for germination. The temperature of this chamber was between 25 and $27{ }^{\circ} \mathrm{C}$ with $90 \%$ relative humidity. Genomic DNA was extracted from 2- to 3-week-old seedlings using a cetyltrimethyl ammonium bromide (CTAB) protocol (Fulton et al. 1995).

\section{Sequence alignment}

The $S$. habrochaites LYC4 sequence from the $T y$ - 2 region was extracted from the draft de novo assembly (The 100 Tomato Genome Sequencing Consortium 2014). Pairwise comparisons between sequences were made using the 
WebAct tool (Abbott et al. 2005) using default settings. Resulting alignments were visualized using the Artemis comparison tool ACT (Carver et al. 2005) with the footprint slider set at 101 (filter the regions of similarity based on the length of sequence over which the similarity occurs). The dot plot was obtained by aligning the $T y-2$ regions from S. lycopersicum 'Heinz' and S. habrochaites LYC4 using MAFFT version 7 (http://mafft.cbrc.jp/alignment/ server/, Katoh and Standley 2013). Sequence analyses were performed with DNASTAR Lasergene 8 and Vector NTIAdvance 11 (Invitrogen).

\section{Recombinant screening}

CAPS markers used for fine-mapping of the $T y-2$ region (Ji et al. 2009; Yang et al. 2014) were tested for polymorphisms between different $S$. habrochaites accessions. PCR products obtained from the different accessions were sequenced, and the sequences were aligned to discover SNPs. When possible, co-dominant CAPS markers were developed to distinguish the different parental alleles. Primer sequences are presented in Table S1. PCRs were performed in 96-wells plates. PCR products were digested with restriction enzymes from Thermo Scientific and New England Biolabs.

\section{Construction and screening of BAC library}

The cultivated tomato line $12 \mathrm{~g}-60$, homozygous for the smallest introgression containing the $T y-2$ resistance gene from Solanum habrochaites B6013, was selected for the construction of a bacterial artificial chromosome (BAC) library. HindIII fragments were cloned into vector CopyControl $^{\mathrm{TM}}$ pCC1BAC ${ }^{\mathrm{TM}}$ (HindIII Cloning-Ready) (Epicentre), and transformed to E. coli strain TransforMax ${ }^{\mathrm{TM}} \mathrm{EPI} 300^{\mathrm{TM}}$ (Epicentre), according to a previously described protocol (Rouppe van der Voort et al. 1999). The BAC library consisted of 99,840 clones with an average insert size of $100 \mathrm{~kb}$, corresponding to 10 times coverage of the tomato genome. The library was stored in 260 384-well microtiter plates, and all 384 clones in one plate were mixed to form a BAC pool. The BAC pool DNA was isolated by alkaline lysis method and screened by PCR using 17 primer pairs within and flanking the $T y-2$ region (Table S2). Afterwards, individual colonies from the 384-well plates corresponding to the positive BAC pools were identified using the same markers, and DNA was isolated from the positive colonies.

\section{DNA sequencing and analysis}

BAC ends were sequenced to confirm that they originated from the $T y-2$ region. Complete sequences of the selected BAC clones (16-100 kb) were obtained by constructing a library of subclones $(1-3 \mathrm{~kb})$. Both ends of the subclones were sequenced using the $\mathrm{ABI} 3730 \mathrm{xl}$ platform and then assembled (BGI, Beijing, China). Putative genes in the BAC sequence were predicted with the online Softberry program FGENESH (Solovyev et al. 2006). Results were compared with the 'Heinz' 1706 genome annotations derived from the International Tomato Annotation Group (ITAG2.3 version). Primers used to analyse the putative inversion breakpoints were bpTyF1 (5'-AAACTCACACCGCTCCGTTGTC-3'), bpTyR1 (5'-CCTCTTCCGATCTTTGGGTACA- $\left.3^{\prime}\right)$ and bpTyR2 (5'-TGTTGGCATGTGACTTATAGGTA-3').

\section{Results}

\section{Bioinformatic comparison of the $T y-2$ region from $S$. lycopersicum and $S$. habrochaites}

The region containing the $T y-2$ resistance gene was determined to span a $300-\mathrm{kb}$ sequence at the distal end of the long arm of chromosome 11, flanked by markers UP8 and M1 (Fig. 1a, Yang et al. 2014). This corresponds to the region between nucleotides 51,344,943 and 51,646,517 on chromosome 11 of tomato genome version SL2.40, or between nucleotides $54,261,443$ and $54,563,017$ on chromosome 11 of tomato genome version SL2.50. We prefer to use the coordinates of the SL2.40 version in this paper for easy reference to previous articles.

A BLAST analysis of this region was performed against the draft de novo assembly of the $S$. habrochaites LYC4 genome. Three large scaffolds (531, 1459 and 770) spanned most of the $T y-2$ region (Fig. 1a). Interestingly, S. habrochaites LYC4 scaffold 531 contained both the flanking marker UP8 and marker C2_At3g52090 at a distance of only $26 \mathrm{~kb}$, whereas in S. lycopersicum 'Heinz' the distance between these two markers is $262 \mathrm{~kb}$. Additionally, S. habrochaites LYC4 scaffold 1459 contained both markers P8-8 and UP15 at a distance of $24 \mathrm{~kb}$, whereas in S. lycopersicum 'Heinz' the distance between these two markers is $247 \mathrm{~kb}$.

The three scaffolds 531, 1459 and 770 of $S$. habrochaites LYC4 were connected to form a superscaffold. To confirm the linkage between the scaffolds PCRs were performed. PCR products spanning the gaps between the scaffolds were obtained and sequenced. The gap between scaffolds 531 and 1459 proved to be small (606 bp, Fig. 1a). The gap between scaffolds 1459 and 770 was larger, approximately $4.3 \mathrm{~kb}$. Thus, by closing the gaps we confirmed the orientation of the three scaffolds.

By aligning the $T y-2$ regions of $S$. lycopersicum 'Heinz' and S. habrochaites LYC4 we observed an inversion of $\pm 200 \mathrm{~kb}$ in the central part (Fig. 1b). Within this inversion there is good co-linearity between 'Heinz' and LYC4, except for some gaps (unknown sequences) in the assemblies, of 
Fig. 1 Comparison between the $T y-2$ genomic region of $S$. lycopersicum 'Heinz' and the superscaffold spanning the Ty-2 region in S. habrochaites LYC4 (a) Visual representation of the $T y$-2 regions in 'Heinz' and LYC4. Markers in the 'suppression of recombination' block are indicated in red (UP15, P1-19, TG36, cL2 and C2_At3g52090), and the other markers are in black (UP8, 51355_MH, P8-8 and M1). Gaps in the 'Heinz' sequence are shown in light blue bars and the sizes of these gaps are estimated by the number of " $\mathrm{N}$ " in the tomato genome. NB-LRR genes are indicated as green arrows. Orange dotted lines connect homologous sequences in LYC4 compared with 'Heinz'. b Dot plot of the alignment of the $T y-2$ regions of 'Heinz' and LYC4. Red lines indicate co-linearity; blue lines indicate inversion. The gaps in the 'Heinz' sequence disrupt the co-linearity of the two sequences
A

S. lyc. Heinz Ty-2 region (301 kb)

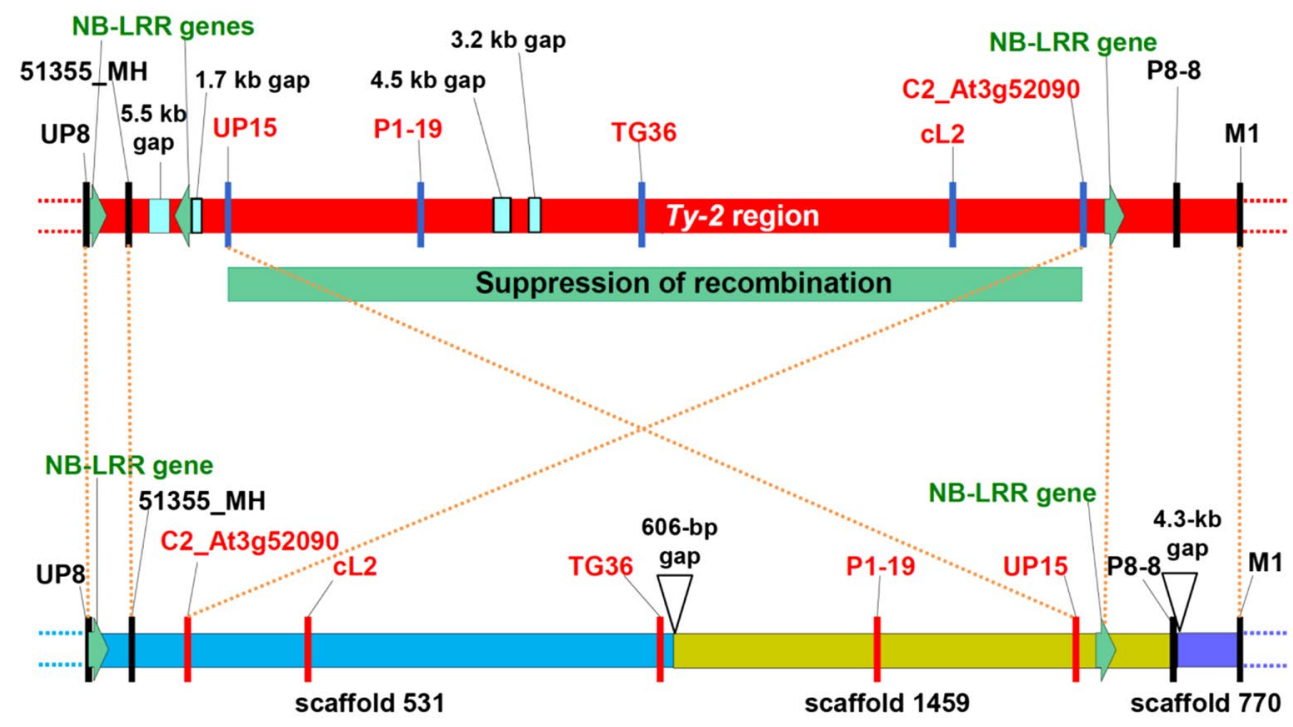

S. habr. LYC4 TY-2 region (278 kb)

B

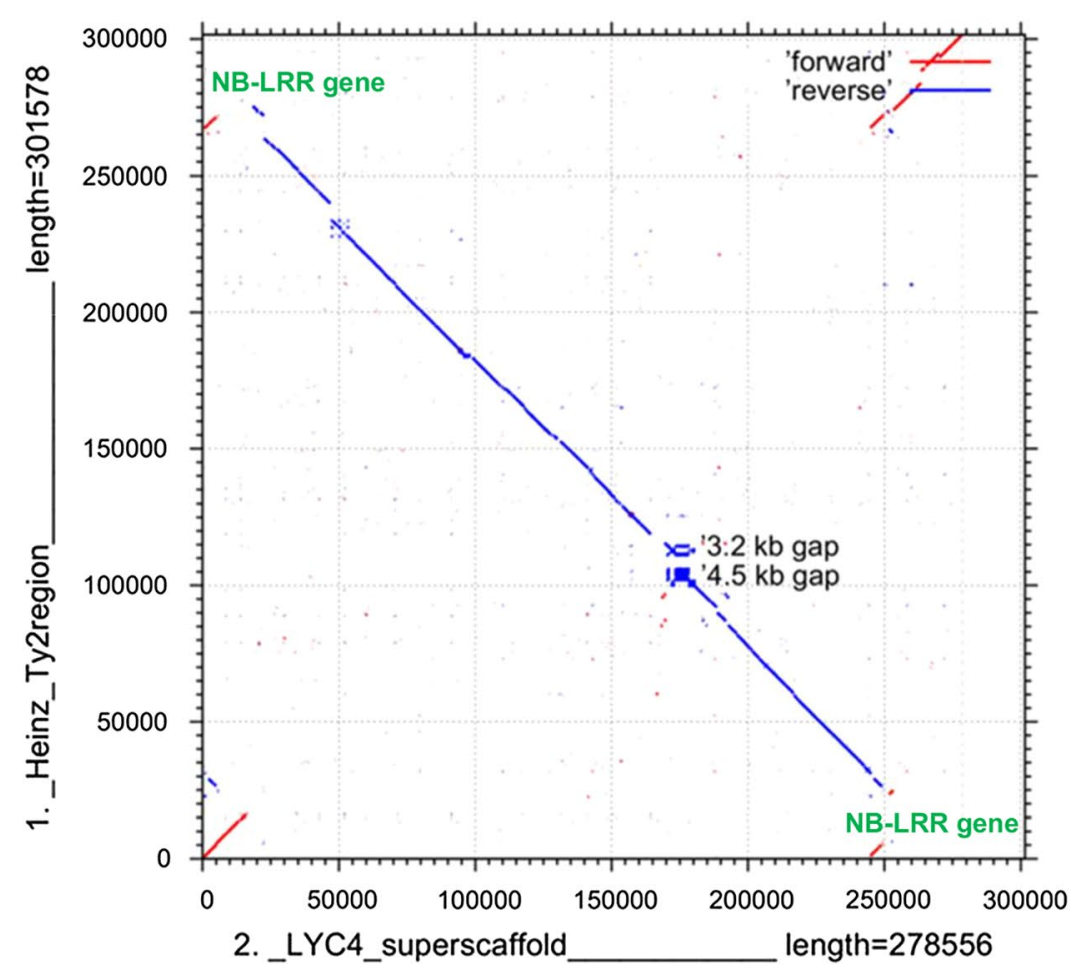

which the largest ones are indicated in Fig. 1a. This inversion coincides with the 'suppression of recombination' block in progeny of the interspecific cross between $S$. lycopsersicum and the Ty-2 donor S. habrochaites B6013 (Yang et al. 2014).

\section{Recombinant screening within $S$. habrochaites species}

Previously, Yang et al. (2014) reported a severe suppression of recombination in the $T y-2$ region in an interspecific cross between $S$. lycopersicum and a $\mathrm{BC}_{4} \mathrm{~S}_{2}$ introgression line derived from $S$. habrochaites $\mathrm{B} 6013$ (donor of the $T y$-2 gene). Among 11,000 $\mathrm{F}_{4}$ plants no recombinants were observed between markers TG36 and C2_At3g52090 (Fig. 1).

We investigated whether suppression of recombination in the $T y-2$ region is also occurring in intraspecific $S$. habrochaites crosses. For this, we analysed $\mathrm{F}_{2}$ populations of three crosses between four different $S$. habrochaites 
Fig. 2 Confirmation of inversion in the $T y-2$ region by genetic analysis. CAPS markers used for recombinant screening of intraspecific $S$. habrochaites $\mathrm{F}_{2}$ populations are indicated. UP8 is included as a reference to delineate the $T y$ - 2 region, but was not used as marker

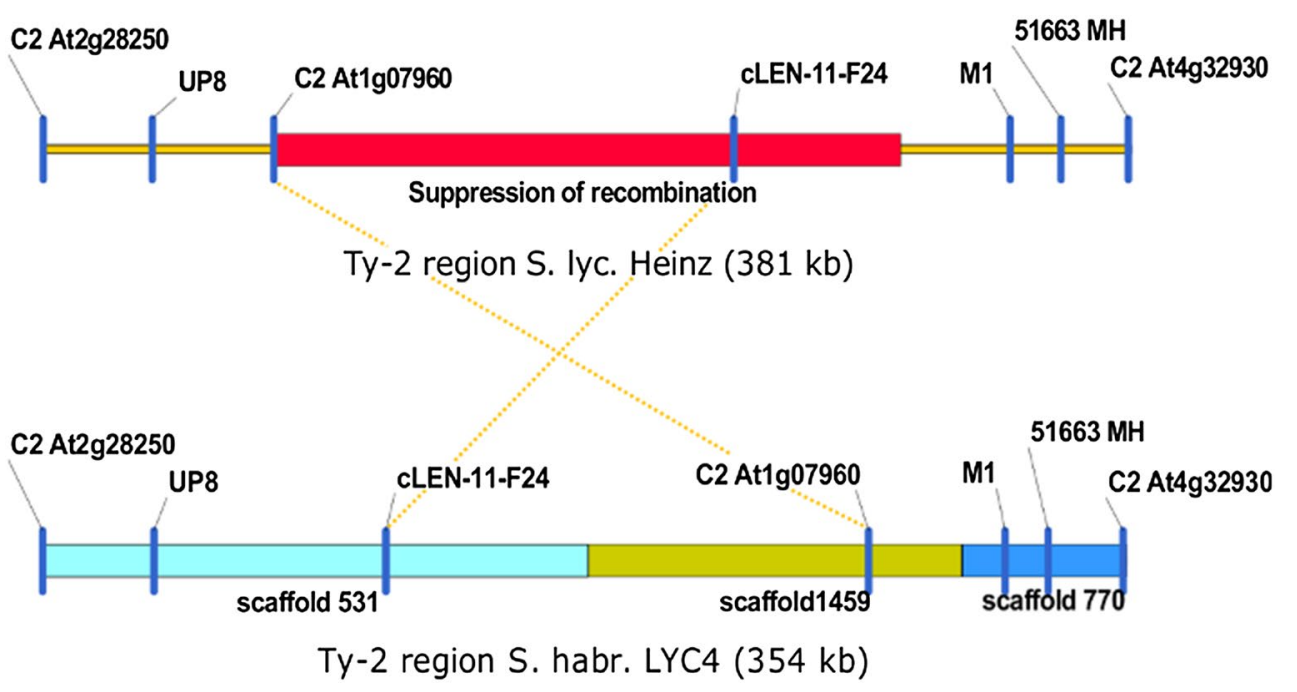

accessions. All crosses had one parent in common, which is accession G1.1560. This accession was chosen as the common parent because it shows a relatively high level of polymorphisms compared with the other three accessions that are more similar to each other. Recombinant screening was performed on 91 to $287 \mathrm{~F}_{2}$ progeny per cross using selected CAPS markers in the $T y-2$ region that had been shown to be polymorphic between G1.1560 and the other S. habrochaites accessions (Fig. 2). These include one marker above the 'suppression of recombination' block (C2_At2g28250), two markers within the block (C2_At1g07960/UF_07960 and cLEN-11-F24), and three markers below the block (M1, 51663_MH and C2_At4g329530). Markers C2_ At1g07960 and UF_07960 are derived from the same gene, but amplify different fragments. Polymorphisms could be detected in one or the other marker, depending on the crossing population.

First, we analysed occurrence of recombination between markers C2_At1g07960/UF_07960 and cLEN-11-F24. The physical distance between these markers in the $S$. lycopersicum 'Heinz' genome is $162 \mathrm{~kb}$, while the genetic distance between these markers is $4.5 \mathrm{cM}$ in the Tomato-EXPEN 2000 genetic map. In total, 21 recombinants were found between these two markers (Table 1), seven in population 1 (PV960357, 91 plants), six in population 2 (PV970303, 287 plants) and eight in population 3 (PV960350, 96 plants). This indicated that there is no suppression of recombination in this region in intraspecific $S$. habrochaites crosses, although the genetic distance between these two markers varies among the crosses $(2-8 \mathrm{cM})$.

To determine marker order in $S$. habrochaites, markers flanking the 'suppression of recombination' block (UP8, C2_At2g28250, M1, 51663_MH and C2_At4g32930) were included in the analysis (Table 1). When the markers are ordered according to the S. lycopersicum 'Heinz' genome three crossovers in a relatively small region of $338 \mathrm{~kb}$ are required to explain the obtained recombinant genotypes. However, a single recombination is sufficient to explain these genotypes when the order of markers C2_At1g07960 and cLEN-11-F24 is reversed. This strongly suggests that an inversion of the region containing these two markers is present in multiple $S$. habrochaites accessions compared with $S$. lycopersicum.

To investigate whether suppression of recombination in the $T y-2$ region is unique to the cross described by Yang et al. (2014) we analysed $88 \mathrm{~F}_{2}$ plants from a different interspecific cross, between $S$. lycopersicum 'Moneymaker' (MM) and TYLCV-susceptible S. habrochaites accession G1.1257 (parent of population 3, PV960350). No recombination events were found between markers C2_At1g07960/ UF_07960 and cLEN-11-F24, suggesting a suppression of recombination in this population.

\section{Analysis of inversion breakpoints}

So far, only a draft version of the de novo assembly of the $S$. habrochaites LYC4 genome is available. Alignment of the LYC4 superscaffold to the 'Heinz' genome sequence showed that the inversion was flanked by NB-LRRlike genes in inverted orientation in the 'Heinz' genome (Fig. 3a). One could argue that the inversion in the LYC4 superscaffold is due to misassembled sequences. To obtain evidence for the presence of an inversion in the $T y-2$ region in S. habrochaites compared with S. lycopersicum, a BAC library was made of a $T y-2$ introgression line. This line contains a small introgression of the $T y-2$ region from $S$. habrochaites 'B6013' (donor of the Ty-2 gene) in an otherwise S. lycopersicum background. A BAC containing the UP15 marker (Fig. 1a) was obtained and sequenced. Alignment of this sequence to the $S$. lycopersicum 'Heinz' sequence (Fig. 3a) showed that a large part was homologous to the upper end of the inversion in S. lycopersicum, which is as 


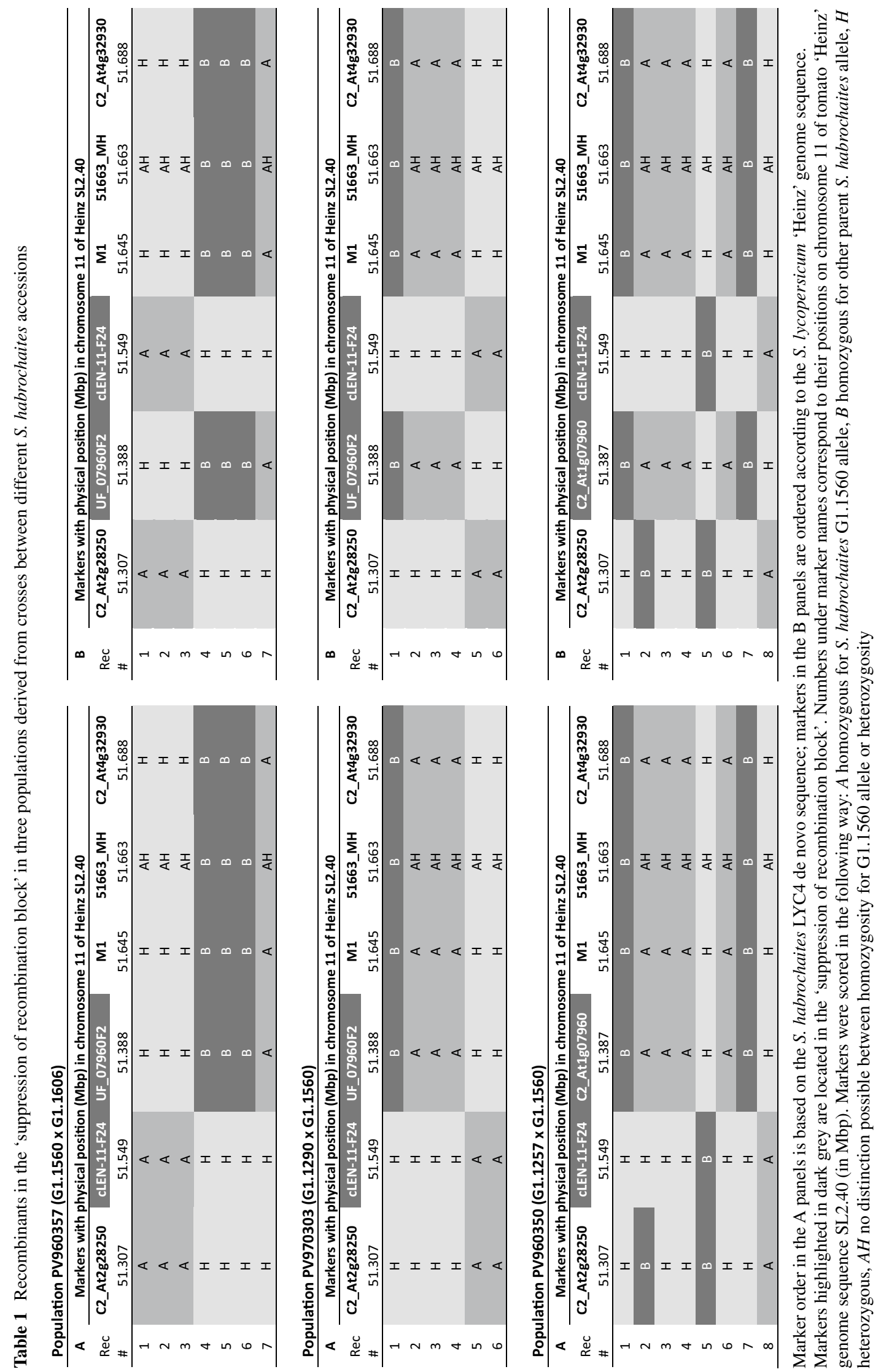




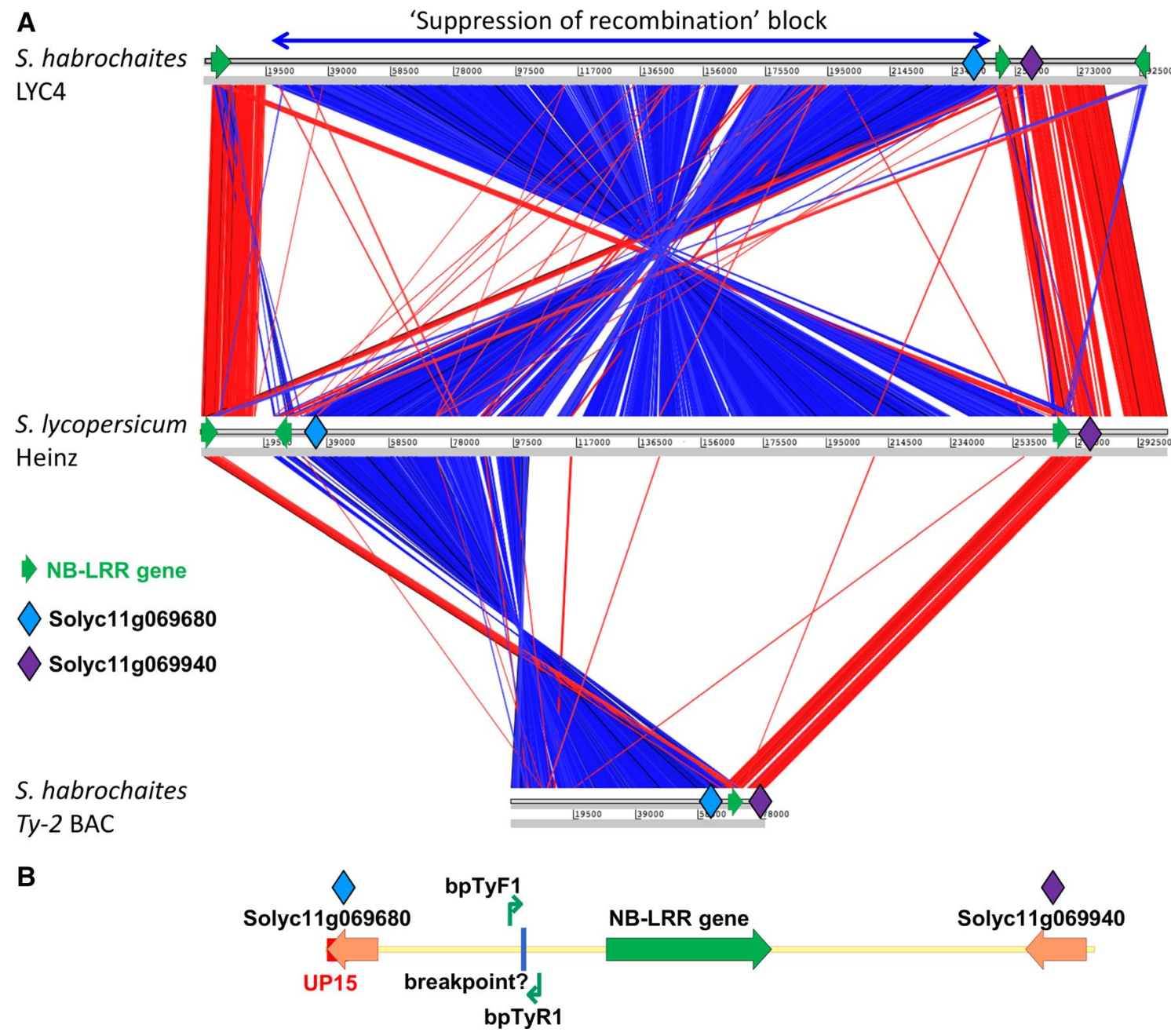

Fig. 3 Comparative sequence analyses (a) Visualization of the alignment of the $S$. habrochaites LYC4 Ty-2 region and the BAC sequence from the $T y-2$ introgression line with the $S$. lycopersicum 'Heinz' Ty-2 region. Red lines indicate homology in the direct orientation $(+$ strand). Blue lines indicate homology in the inverse orientation ( - strand). The blue lines indicate the presence of an inversion when comparing $S$. habrochaites with $S$. lycopersicum. NB-LRR gene

expected based on the location of the UP15 marker which is derived from gene Solyc11g069680 (Fig. 3b). However, it additionally contained a sequence homologous to gene Solyc11g069940, which is close to M1 (Fig. 1), a marker at the other side of the inversion. Thus, the BAC contained predicted genes homologous to Solyc11g069680 and Solyc11g069940 in close proximity (18 kb) (Figure S1). In between these genes an NB-LRR type of gene is predicted that shows homology to both Solyc11g069660 and Solyc11g069930.

A detailed analysis of the BAC sequence was performed to determine the location of the inversion breakpoint (Fig. 4a). Primers flanking the potential breakpoint were designed on the $T y-2$ BAC sequence (Figs. 3b, 4a). sequences (indicated as green arrows) align to different positions in both direct and inverse orientation. Positions of genes homologous to Solyc11g06980 and Solyc11g069940 are indicated. b Part (18 kb) of the $T y-2$ BAC containing the lower inversion breakpoint. Primers (bptyF1 and bpTyR1) spanning the putative inversion breakpoint are indicated

They amplified a 732-bp fragment in the $T y-2$ introgression line (Fig. 4b). As expected, no PCR product was obtained with $S$. lycopersicum MM DNA. In the 'Heinz' sequence the reverse primer bpTyR1 is present in the same orientation as in the $T y-2 \mathrm{BAC}$ sequence, while the forward primer bpTyF1 is present in the inverse orientation. However, reverse primer bpTyR1 is also present on the other side of the inversion in 'Heinz', between markers 51355_MH and UP15. A PCR product of approximately $8.9 \mathrm{~kb}$ might be obtained if the adequate PCR conditions for long PCR products would be applied. Remarkably, also no PCR product was obtained for $S$. habrochaites LYC4 (Fig. 4b). When comparing the $T y-2$ BAC sequence with the LYC4 superscaffold sequence we found that the forward primer 


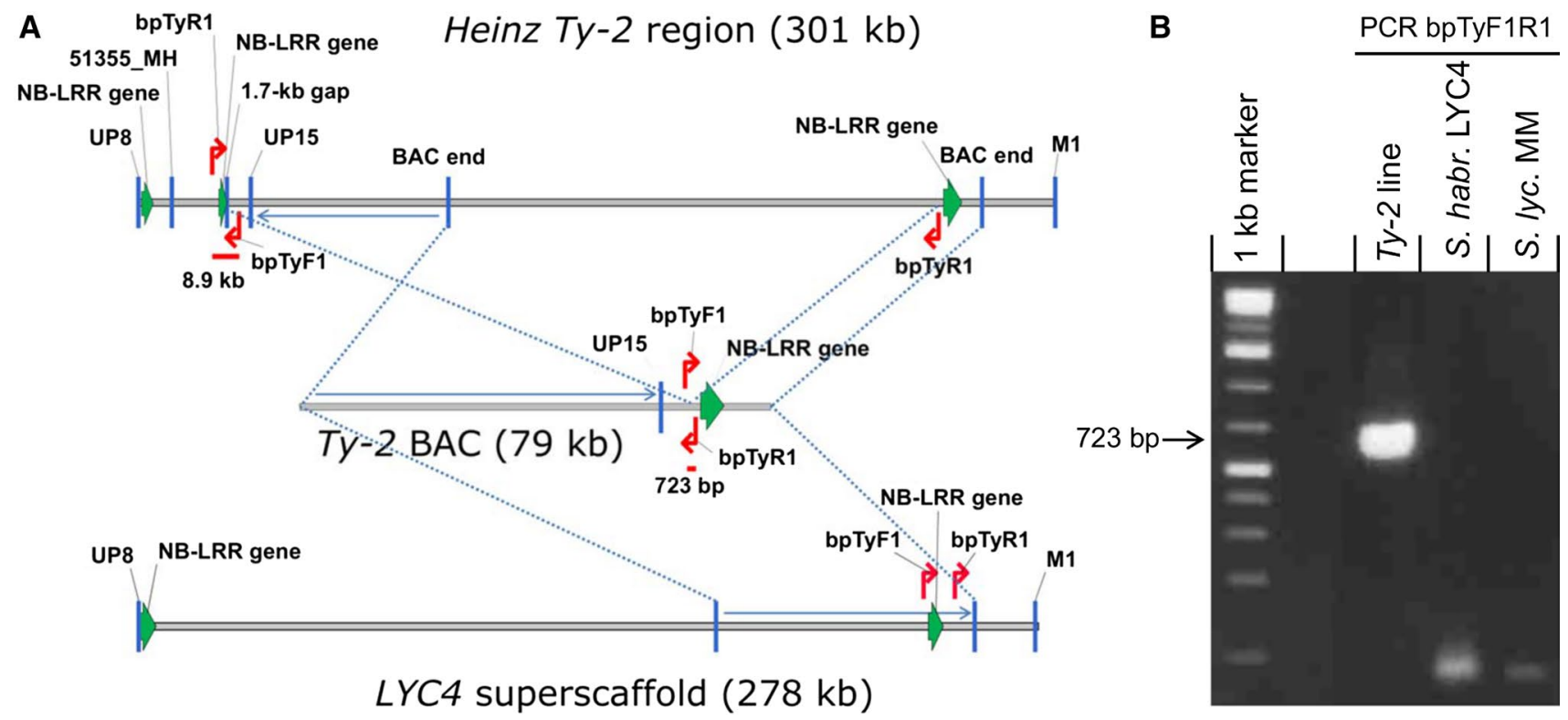

Fig. 4 Analysis of inversion breakpoint (a) Location of breakpoint primers bpTyF1 and bpTyR1 (red arrows) flanking the inversion breakpoint in the BAC sequence obtained from the $T y-2$ introgression line (middle sequence). Location of these primers is also shown in the S. lycopersicum 'Heinz' region (upper sequence) and S. habro-

bpTyF1 was in the expected position above an NB-LRR gene (Fig. 4a). However, the reverse primer bpTyR1 was present below the NB-LRR gene, in the same orientation as the forward primer bpTyF1. This explains why no amplification product was obtained for LYC4.

The 732-bp sequence of the PCR product obtained from the $T y$-2 introgression line (Fig. $4 \mathrm{~b}$ ) was aligned with the sequence upstream of the unique primer bpTyF1 in the 'Heinz' genome (Figure S1A). These sequences showed a poor alignment, except for the first $154 \mathrm{bp}$ starting from primer bpTyF1. In order to verify this breakpoint region in cultivated tomato, primer bpTyR2 was developed based on the 'Heinz' genome sequence (Figure S1B). A PCR with primers bpTyF1 and bpTyR2 resulted in the expected 687-bp product in $S$. lycopersicum MM but not in the $T y-2$ introgression line (Figure S1C). The sequence of the 687bp PCR product of MM was identical to the sequence in 'Heinz'. Although the alignment of the sequences of the two PCR products show an abrupt end of co-linearity, it is preliminary to conclude that this is the exact breakpoint of the inversion. To verify this conclusion, we need to know the sequence of the upper breakpoint region in the $T y$-2 introgression line. Figure S1B shows the regions harbouring the upper and lower breakpoints in S. lycopersicum 'Heinz', and the lower breakpoint in the BAC clone from the $T y-2$ introgression line. Gene Solyc11g069680 is present in the upper breakpoint region, while gene Solyc11g069940 is present in the lower breakpoint region chaites LYC4 superscaffold (lower sequence). Green arrows indicate sequences homologous to NB-LRR genes. Blue dotted lines indicate co-linear regions. b PCR with primers bpTyF1 and bpTyR1 flanking the inversion breakpoint. Only in the $T y-2$ introgression line a PCR product is obtained

in the 'Heinz' genome. Both genes are adjacent to NBLRR gene fragments. The $T y-2$ BAC sequence contains orthologs of both Solyc11g069680 and Solyc11g069940, separated by a NB-LRR gene.

\section{Discussion}

\section{Chromosomal rearrangements are frequently associated with resistance gene clusters}

We report the presence of an inversion of a $\pm 200 \mathrm{~kb}$ region on the long arm of chromosome 11 in $S$. habrochaites compared with S. lycopersicum. This inversion is different from the 294-kb inversion underlying the fasciated locus on the long arm of chromosome 11, which is polymorphic within the cultivated S. lycopersicum germplasm (Huang and Van der Knaap 2011).

There are numerous examples of chromosomal rearrangements/inversions associated with (introgression of) R-genes or R-gene clusters. Two R-gene clusters in the $\mathrm{Mi}$ locus on the short arm of chromosome 6 in Solanum peruvianum are separated by approximately $300 \mathrm{~kb}$ region, which is inverted compared to S. lycopersicum (Seah et al. 2007). The introgression of the $T y-1$ locus on the long arm of chromosome 6 from $S$. chilense in S. lycopersicum background shows an inversion and suppression of recombination (Verlaan et al. 2011). The Hl locus on the distal end 
of chromosome 5 of potato (Finkers-Tomczak et al. 2011) shows repression of recombination in a region of at least $170 \mathrm{~kb}$. The $R I$ locus in the same region was shown to be present in a region that was inverted in tomato compared with potato (Achenbach et al. 2010). The donor of the $R I$ gene, $S$. demissum, contained haplotypes that were highly diverged in the R-gene cluster region, while the flanking non-resistance gene regions were conserved (Kuang et al. 2005). A 70-kb inversion between the resistant R1 and the susceptible $\mathrm{r} 1$ haplotypes was reported by Ballvora et al. (2007). The clubroot resistance region in Brassica rapa has an internal inversion compared with Arabidopsis of about $310 \mathrm{~kb}$ (Suwabe et al. 2012). Suppression of recombination in these R-gene regions may be a consequence of the chromosomal rearrangement. On the other hand, suppressed recombination may also be caused by the pericentromeric position of the introgression rather than the inversion, as is the case for the Mi-l locus (Seah et al. 2007).

For other resistance gene loci suppressed recombination has been reported, but it is unknown whether this is a consequence of chromosomal rearrangements, and/or of pericentromeric locations. These include the $T m-2 a$ gene from $S$. peruvianum introgressed in S. lycopersicum (Pillen et al. 1996), the MXC3 gene in poplar, the Lr20-Sr15-Pml resistance locus and $\operatorname{Sr} 22, \operatorname{Lr} 9, \operatorname{Lr} 24$ and $L r 35$ resistance genes in wheat, the Mla and Mlg powdery mildew resistance gene clusters and the Rrs2 resistance gene in barley (reviewed in Hanemann et al. 2009), and the Rhgl/Rfs2 locus in soybean (Afzal et al. 2012).

Chromosome rearrangements complicate the fine-mapping and cloning of resistance genes, especially when they involve large regions containing many genes. In the case of the $T y-2$ resistance gene it was shown previously that it is unlikely to be a typical NB-LRR gene, because silencing of the NB-LRR candidates in the $T y$ - 2 region did not result in compromised TYLCV resistance (Yang et al. 2014).

\section{Advantage of de novo genome assemblies of wild relatives of crop species}

After the assembly of the genome of cultivated crop species the focus has shifted to sequencing related wild species at low read depth to obtain information on sequence variation by mapping reads to the reference genome. The assumption is that there is a high degree of co-linearity within a species and between closely related species, and that a large set of SNP markers developed after re-sequencing can be used to fine map traits of interest. However, as shown by Huang and van der Knaap (2011) chromosomal rearrangements may occur even within a cultivated species. Re-sequencing data consisting of small reads do not provide positional information of SNP markers, or SNP marker order. Therefore, such data do not uncover the presence of chromosomal rearrangements in wild species, especially those that are not closely related to the cultivated species as shown in tomato (Szinay et al. 2012). FISH using BAC clones has been demonstrated to be a powerful tool in the study of chromosomal rearrangements (Lou et al. 2010; Verlaan et al. 2011; Peters et al. 2012; Szinay et al. 2012; Shearer et al. 2014). However, in the $T y-2$ region, FISH was not successful due to the small size of the inversion (Yang et al. 2014). In this study, we show that a de novo genome assembly has been very helpful to analyse the chromosomal structure of a wild species, which can be exploited to explain unexpected recombination phenomena in crosses with the cultivated species.

Also within a wild species there may be accessions that show small-scale rearrangements, as we observed when comparing the inversion breakpoint between $S$. habrochaites LYC4 and the Ty-2 BAC sequence derived from S. habrochaites B6013. Therefore, BAC libraries may still be required to zoom in on the gene of interest in specific accessions.

\section{Perspectives for resistance gene cloning}

Introgression of the smallest possible DNA fragment containing the gene of interest from a donor species into the crop species is often a time-consuming process, and the success can be limited when chromosomal rearrangements exist in related species used for interspecific crosses. Since genome structure and genomic co-linearity of the introgressed region between donor species and recipient crops are often unknown, breeders are 'blind' and cannot foresee complications in their introgression breeding programs. With the example of the $T y-1$ gene (Verlaan et al. 2011) and the $T y-2$ gene in this study, we demonstrated that FISH and genomic approaches can be applied to investigate chromosomal rearrangements in genetic mapping and introgression breeding. Furthermore, the occurrence of chromosomal rearrangements stresses the importance of a de novo genome assembly when wild Solanum species are sequenced.

The fact that $T y-2$ is located in a chromosomal region which is inverted in S. habrochaites compared with S. lycopersicum has consequences for the strategy of cloning this gene. For marker-assisted breeding it is not necessary to clone the gene conferring resistance to TYLCV, because linked markers in the 'suppression of recombination' block do not show segregation in the progeny. However, this large block introgressed from $S$. habrochaites contains at least 35 genes (Yang et al. 2014), of which it is unknown whether they have an adverse effect on plant growth, performance and yield in diverse growing conditions. Negative effects on agronomic and quality traits have been observed to be associated with introgression from the $T m-2 a, S w-5$ and $T y$ 1 virus resistance genes (Rubio et al. 2012), probably due to linkage drag. 
Here, we show that recombination in the $T y$ - 2 region is occurring in intraspecific crosses between different $S$. habrochaites accessions. Therefore, in order to further fine-map the TYLCV resistance gene we are generating $\mathrm{F}_{2}$ progenies from a cross between resistant $S$. habrochaites accession B6013 and susceptible S. habrochaites accessions that show enough polymorphisms for efficient and detailed recombinant screening. In the near future, the finemapped position of the $T y-2$ gene will show whether it is located in the inversion. If the gene is outside the inversion, it should be possible to eliminate the inversion in an introgression line carrying the $T y-2$ gene.

Author contribution statement AMAW, YD and YB designed the experiments; AMAW, MC, SD, RF, JG and $\mathrm{XW}$ performed the experiments; AMAW, MC and YB analysed the data. AMAW, SD and YB wrote the paper; RGFV critically read and improved the paper.

Acknowledgments We thank Marc Hendriks, Anindita Chakraborty, Sayantani Chatterjee and Yucheng Huang for their assistance in marker analysis. This project was partially funded by the Netherlands Top Consortium for Knowledge and Innovation (TKI project TU-370), the National Natural Science Foundation of China (31372070), The Major Project of Chinese National Programs for Fundamental Research and Development (2011CB100600) and an internal grant of Wageningen UR Plant Breeding.

Conflict of interest The authors declare that they have no conflict of interest.

Open Access This article is distributed under the terms of the Creative Commons Attribution 4.0 International License (http://creativecommons.org/licenses/by/4.0/), which permits unrestricted use, distribution, and reproduction in any medium, provided you give appropriate credit to the original author(s) and the source, provide a link to the Creative Commons license, and indicate if changes were made.

\section{References}

Abbott JC, Aanensen DM, Rutherford K, Butcher S, Spratt BG (2005) WebACT - an online companion for the artemis comparison tool. Bioinformatics 21:3665-3666

Achenbach UC, Tang X, Ballvora A, de Jong H, Gebhardt C (2010) Comparison of the chromosome maps around a resistance hot spot on chromosome 5 of potato and tomato using BAC-FISH painting. Genome 53:103-110

Afzal AJ, Srour A, Saini N, Hemmati N, El Shemy HA, Lightfoot DA (2012) Recombination suppression at the dominant Rhg1/Rfs 2 locus underlying soybean resistance to the cyst nematode. Theor Appl Genet 124:1027-1039

Anderson LK, Covey PA, Larsen LR, Bedinger P, Stack SM (2010) Structural differences in chromosomes distinguish species in the tomato clade. Cytogenet Genome Res 129:24-34

Ballvora A, Jöcker A, Viehöver P, Ishihara H, Paal J, Meksem K, Bruggmann R, Schoof H, Weisshaar B, Gebhardt C (2007) Comparative sequence analysis of Solanum and Arabidopsis in a hot spot for pathogen resistance on potato chromosome $\mathrm{V}$ reveals a patchwork of conserved and rapidly evolving genome segments. BMC Genom 8:112

Carver TJ, Rutherford KM, Berriman M, Rajandream MA, Barrell BG, Parkhill J (2005) ACT: the Artemis comparison tool. Bioinformatics 21:3422-3423

Doğanlar S, Frary A, Daunay MC, Huvenaars K, Mank R, Frary A (2014) High resolution map of eggplant (Solanum melongena) reveals extensive chromosome rearrangement in domesticated members of the Solanaceae. Euphytica 198:231-241

Finkers-Tomczak A, Bakker E, de Boer J, van der Vossen E, Achenbach U, Golas T, Suryaningrat S, Smant G, Bakker J, Goverse A (2011) Comparative sequence analysis of the potato cyst nematode resistance locus $H 1$ reveals a major lack of co-linearity between three haplotypes in potato (Solanum tuberosum ssp.). Theor Appl Genet 122:595-608

Fulton TM, Chunwongse J, Tanksley SD (1995) Microprep protocol for extraction of DNA from tomato and other herbaceous plants. Plant Mol Biol Rep 13:207-209

Hanemann A, Schweizer GF, Cossu R, Wicker T, Röder MS (2009) Fine mapping, physical mapping and development of diagnostic markers for the Rrs 2 scald resistance gene in barley. Theor Appl Genet 119:1507-1522

Hanson PM, Bernacchi D, Green S, Tanksley SD, Muniyappa V, Padmaja AS, Chen H, Kuo G, Fang D, Chen J (2000) Mapping a wild tomato introgression associated with tomato yellow leaf curl virus resistance in a cultivated tomato line. J Am Soc Hort Sci 125:15-20

Huang Z, van der Knaap E (2011) Tomato fruit weight 11.3 maps close to fasciated on the bottom of chromosome 11. Theor Appl Genet 123:465-474

Iovene M, Wielgus SM, Simon PW, Buell CR, Jiang J (2008) Chromatin structure and physical mapping of chromosome 6 of potato and comparative analysis with tomato. Genetics 180:1307-1317

Ji Y, Scott JW, Schuster DJ (2009) Toward fine mapping of the Tomato Yellow Leaf Curl Virus resistance gene Ty-2 on chromosome 11 of tomato. Hort Sci 44:614-618

Kalloo, Banerjee MK (1990) Transfer of tomato leaf curl virus resistance from Lycopersicon hirsutum f. glabratum to L. esculentum. Plant Breeding 105:156-159

Katoh K, Standley DM (2013) MAFFT multiple sequence alignment software version 7: improvements in performance and usability. Mol Biol Evol 30:772-780

Kuang H, Wei F, Marano MR, Wirtz U, Wang X, Liu J, Shum WP, Zaborsky J, Tallon LJ, Rensink W, Lobst S, Zhang P, Tornqvist CE, Tek A, Bamberg J, Helgeson J, Fry W, You F, Luo MC, Jiang J, Buell RC, Baker B (2005) The R1 resistance gene cluster contains three groups of independently evolving, type I $R I$ homologues and shows substantial variation among haplotypes of Solanum demissum. Plant J 44:37-51

Lou Q, Iovene M, Spooner DM, Buell CR, Jiang J (2010) Evolution of chromosome 6 of Solanum species revealed by comparative fluorescence in situ hybridization mapping. Chromosoma 119:435-442

Peters SA, Bargsten JW, Szinay D, van de Belt J, Visser RGF, Bai Y, de Jong H (2012) Structural homology in the Solanaceae: analysis of genomic regions in support of synteny studies in tomato, potato and pepper. Plant J 71:602-614

Pillen K, Ganal MW, Tanksley SD (1996) Construction of a highresolution genetic map and YAC-contigs in the tomato Tm-2a region. Theor Appl Genet 93:228-233

Rouppe van der Voort J, Kanyuka K, van der Vossen E, Bendahmane A, Mooijman P, Klein-Lankhorst R, Stiekema W, Baulcombe D, Bakker J (1999) Tight physical linkage of the nematode resistance gene Gpa2 and the virus resistance gene $R x$ on a single segment introgressed from the wild species Solanum tuberosum 
subsp. andigena CPC 1673 into cultivated potato. Mol PlantMicrobe Interact 12:197-206

Rubio F, García-Martínez S, Alonso A, Grau A, Valero M, Ruiz JJ (2012) Introgressing resistance genes into traditional tomato cultivars: effects on yield and quality. Acta Hort 935:29-33

Seah S, Telleen AC, Williamson VM (2007) Introgressed and endogenous $\mathrm{Mi}-1$ gene clusters in tomato differ by complex rearrangements in flanking sequences and show sequence exchange and diversifying selection among homologues. Theor Appl Genet 114:1289-1302

Shearer LA, Anderson LK, de Jong H, Smit S, Goicoechea JL, Roe BA, Hua A, Giovannoni JJ, Stack SM (2014) Fluorescence in situ hybridization and optical mapping to correct scaffold arrangement in the tomato genome. Genes Genomes Genet 4:1395-1405

Solovyev V, Kosarev P, Seledsov I, Vorobyev D (2006) Automatic annotation of eukaryotic genes, pseudogenes and promoters. Genome Biol 7(Suppl 1):1-12

Suwabe K, Suzuki G, Nunome T, Hatakeyama K, Mukai Y, Fukuoka $\mathrm{H}$, Matsumoto S (2012) Microstructure of a Brassica rapa genome segment homoeologous to the resistance gene cluster on Arabidopsis chromosome 4. Breed Sci 62:170-177

Szinay D, Bai Y, Visser R, de Jong H (2010) FISH applications for genomics and plant breeding strategies in tomato and other Solanaceous crops. Cytogenet Genome Res 129:199-210

Szinay D, Wijnker E, van den Berg R, Visser RGF, de Jong H, Bai Y (2012) Chromosome evolution in Solanum traced by cross-species BAC-FISH. New Phytol 195:688-698

Tang X, Szinay D, Lang C, Ramanna MS, van der Vossen EAG, Datema E, Klein Lankhorst R, de Boer J, Peters SA, Bachem C, Stiekema W, Visser RGF, de Jong H, Bai Y (2008) Cross-species bacterial artificial chromosome-fluorescence in situ hybridization painting of the tomato and potato chromosome 6 reveals undescribed chromosomal rearrangements. Genetics 180:1319-1328
The 100 Tomato Genome Sequencing Consortium (2014) Exploring genetic variation in the tomato (Solanum section Lycopersicon) clade by whole-genome sequencing. Plant J 80:136-148

The Potato Genome Sequencing Consortium (2011) Genome sequence and analysis of the tuber crop potato. Nature 475:189-195

The Tomato Genome Consortium (2012) The tomato genome sequence provided insights into fleshy fruit tomato. Nature 485:635-641

van der Knaap E, Sanyal A, Jackson SA, Tanksley SD (2004) Highresolution fine mapping and fluorescence in situ hybridization analysis of sun, a locus controlling tomato fruit shape, reveals a region of the tomato genome prone to DNA rearrangements. Genetics 168:2127-2140

Verlaan MG, Szinay D, Hutton SF, de Jong H, Kormelink R, Visser RGF, Scott JW, Bai Y (2011) Chromosomal rearrangements between tomato and Solanum chilense hamper mapping and breeding of the TYLCV resistance gene $T y-1$. Plant $\mathbf{J}$ 68:1093-1103

Verlaan MG, Hutton SF, Ibrahem RM, Kormelink R, Visser RGF, Scott JW, Edwards JD, Bai Y (2013) The tomato yellow leaf curl virus resistance genes $T y-1$ and $T y-3$ are allelic and code for DFDGD-class RNA-dependent RNA polymerases. PLoS Genet 9:e1003399

Wu F, Tanksley SD (2010) Chromosomal evolution in the plant family Solanaceae. BMC Genom 11:182

Yang X, Caro M, Hutton SF, Scott JW, Guo Y, Wang X, Rashid MH, Szinay D, de Jong H, Visser RGF, Bai Y, Du Y (2014) Fine mapping of the tomato yellow leaf curl virus resistance gene $T y-2$ on chromosome 11 of tomato. Mol Breed 34:749-760 